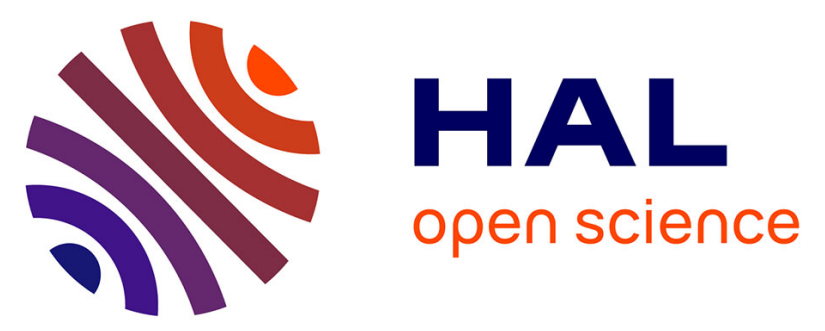

\title{
Alignment of Rod-Shaped Single-Photon Emitters Driven by Line Defects in Liquid Crystals
}

Laurent Pelliser, Mathieu Manceau, Clotilde Lethiec, Delphine Coursault, Stefano Vezzoli, Godefroy Leménager, Laurent Coolen, Massimo Devittorio, Ferruccio Pisanello, Luigi Carbone, et al.

\section{To cite this version:}

Laurent Pelliser, Mathieu Manceau, Clotilde Lethiec, Delphine Coursault, Stefano Vezzoli, et al.. Alignment of Rod-Shaped Single-Photon Emitters Driven by Line Defects in Liquid Crystals. Advanced Functional Materials, 2015, 25 (11), pp.1719-1726 10.1002/adfm.201403331 . hal-01211308

\author{
HAL Id: hal-01211308 \\ https://hal.science/hal-01211308
}

Submitted on 6 Oct 2015

HAL is a multi-disciplinary open access archive for the deposit and dissemination of scientific research documents, whether they are published or not. The documents may come from teaching and research institutions in France or abroad, or from public or private research centers.
L'archive ouverte pluridisciplinaire HAL, est destinée au dépôt et à la diffusion de documents scientifiques de niveau recherche, publiés ou non, émanant des établissements d'enseignement et de recherche français ou étrangers, des laboratoires publics ou privés. 


\section{Alignment of Rod-Shaped Single Photon Emitters Driven by Line Defects in Liquid Crystals}

Laurent Pelliser ${ }^{1,2}$, Mathieu Manceau ${ }^{3}$, Clotilde Lethiec ${ }^{1,2}$, Delphine Coursault ${ }^{1,2}$, Stefano Vezzoli ${ }^{3,4}$, Godefroy Leménager ${ }^{3}$, Laurent Coolen ${ }^{1,2}$, Massimo DeVittorio ${ }^{5,6,7}$, FerruccioPisanello ${ }^{6}$, Luigi Carbone $^{7}$, Agnes Maitre ${ }^{1,2}$, Alberto Bramati ${ }^{3}$ and Emmanuelle Lacaze $e^{1,2 *}$.

${ }^{1}$ CNRS, UMR 7588, Institut des NanoSciences de Paris (INSP), 4 place Jussieu, F-75005 Paris, France.

${ }^{2}$ Sorbonne universités, UPMC Univ Paris 06, UMR 7588, Institut des NanoSciences de Paris (INSP), 4 place Jussieu, F-75005 Paris, France

${ }^{3}$ Laboratoire Kastler Brossel, UPMC-Sorbonne Universités, CNRS, ENS-PSL ResearchUniversity, Collège de France, 4 place Jussieu Case 74, F-75005 Paris, France,

${ }^{4}$ Centre for Disruptive Photonic Technologies, Nanyang Technological University, 637371, Singapore

${ }^{5}$ DipartimentodiIngegneriadell'Innovazione, Università del Salento, via per Monteroni, 73100 Lecce, Italy,

${ }^{6}$ InstitutoItalianodiTecnologia (IIT), Center for Bio-Molecular Nanotechnologies, Via Barsanti, 73010 Arnesano (Lecce), Italy 


\footnotetext{
${ }^{7}$ National Nanotechnology Laboratory (NNL), CNR-IstitutoNanoscienze, Via Arnesano km 5, 73100 Lecce, Italy
}

Keywords: liquid crystals, nanorods, fluorescence, self-alignment, oily streaks

We use arrays of liquid crystal defects, linear smectic dislocations, to trap semi-conductor $\mathrm{CdSe} / \mathrm{CdS}$ dot-in-rods which behave as single photon emitters. We combine measurements of the emission diagram together with measurements of the emitted polarization of the single emitters. We show that the dot-inrods are confined parallel to the linear defects to allow for a minimization of the disorder energy associated with the dislocation cores. We demonstrate that the electric dipoles associated with the dotin-rods, tilted with respect to the rods, remain oriented in the plane including the smectic linear defects and being perpendicular to the substrate, most likely due to the dipole/dipole interactions between the dipoles of the liquid crystal molecules and the dot-in-rods ones. Using smectic dislocations, we can consequently orient nanorods along a unique direction for a given substrate, independently of the ligands' nature, without any induced aggregation, leading as well to a fixed azimuthal orientation for the associated dot-in-rods' dipoles. These results open the way for a fine control of nanoparticle anisotropic optical properties, in particular a fine control of single photon emission polarization.

Control of single photon emitters is a major objective in the field of nanophotonics. ${ }^{[1]}$ The synthesis of colloidal semiconductor inorganic nanocrystals having specific light-emission properties has been providing important advances in this field. In particular, recent developments in synthesis methodologies, fully compatible with standard nanofabrication technologies have enabled a superior 
control on nanocrystals composition and morphology.Rod-shaped nanocrystals showing pronounced polarization, behaving as emitting linear dipoles, have been obtained ${ }^{[2-4]}$ The encapsulation of a spherical core into a rod-like shell ${ }^{[5]}$ resulted in non-blinking inorganic single photon emitters, ${ }^{[6]}$ hereafter referred to as dot-in-rods (DRs). Moreover it has been recently shown that, by increasing the thickness of the shell, it is possible to greatly suppress photoluminescence blinking and to improve DRs overall photo-stability, while keeping a low probability of multi-photon emission. ${ }^{[7]}$ Such features are of primary importance when nanocrystalsare used in applications demanding a control of photons'polarization, such as coupling with complex photonic cavities ${ }^{[8-9]}$ or quantum cryptography. ${ }^{[10]}$ The control of the polarization of the emitted light also requires the capacity to control the particle orientation. Howevertechnologies aimed at guiding nanocrystal orientation at the single particle level are still poorly discussed in literature.

Alignednanoparticleshave been obtained through mechanical rubbing, ${ }^{[11]}$ short-range interactions ${ }^{[12-13]}$ or patterned substrates. ${ }^{[14]}$ Liquid crystal-like structures, composed of alarge number of elongated nanocrystalsassembled in multi-layers have also been evidenced on both solid substrates ${ }^{[15-18]}$ and water films. ${ }^{[18-20]}$ Orientation and positional ordering of CdS and CdSenanorods has been obtained through the utilization of a local electric field, exploiting their intrinsic electric dipole moments, ${ }^{[18,21-23]}$. However, single nanorods are rarely observed once aligned onto the substrate: only average optical properties can be inferred from these experiments. The fluorescence polarization of a number of single DRshas been measured but not for aligned ones. ${ }^{[5,6,24,25]}$ In order to obtain a macroscopic organization and orientation ofsingle nanorods onto a substrate, the use of anisotropic matrices working as hard or soft templatesoffers a promising experimental alternative, as shown for polymer matrices ${ }^{[26-27]}$, which can be stretched ${ }^{[5,28]}$,as for DNA molecules ${ }^{[29]}$ andcarbonnanotubes. ${ }^{[30]}$ 
An increasing number of works is alsodevoted to the use of anisotropic matrices made ofliquid crystals. ${ }^{[31-36]}$ Cholesteric liquid crystals have been used to control the circular polarization of single quantum dots. ${ }^{[37]}$ Nematic liquid crystals have been used to orient single dye molecules, either parallel or perpendicular to the nematic director, depending on the molecular shape ${ }^{[37,38]}$ In nematic liquid crystals,nanorods can be oriented as well, either parallel or perpendicular to the liquid crystal director,depending on the liquid crystal ability to anchor the nanorod surface, ${ }^{[39,40]}$ with the possible help of magnetic fields. ${ }^{\left[41,{ }^{42}\right.}$ However, a serious drawback met in the use of most of thermotropicliquid crystals corresponds to the induced aggregation of thenanorods. ${ }^{[36,43-44]}$ This aggregation is due to the distortions and disorder induced in the liquid crystal around most of the nanoparticles, which become reduced if the nanoparticles are aggregated ${ }^{[36,45]}$ In nematic liquid crystals, the best method to prevent aggregation of nanorods is to graft specific ligandsaround the nanorodsin order to allow for a weak anchoring of the liquid crystal molecules. ${ }^{[46,47]}$ Weak anchoring indeed prevents the formation of liquid crystal defects and thus prevents aggregation. For semiconductor nanorods embedded in liquid crystals, average orientations parallel to the nematic director have been evidenced but, to the best of our knowledge, again only ensemble anisotropic emission properties have been studied. ${ }^{[22,46,48-49]}$

Herein we demonstrate that linear arrays of smecticliquid crystal defects behave as a "smart" matrix to govern the positional and directional ordering of nanorods. Single nanorods can be manipulated and oriented along the defects, along a unique direction for a given substrate. This avoids any liquid crystalinduced aggregation of nanorods, independently of the ligand's nature. We evidence the phenomenon withDRs, single photon emitters,at the single particle level. Statistical analysis of the in-plane orientation of the single DRs leads to a unique orientation with $8^{\circ}$ of standard deviation, leading therefore to a fine control of single photon emission polarization. 
Although we use core-shell CdSe/CdSDRs as a model system, the technology can be easily extended to a wide class of anisotropic nano-objects. The choice of these particles was dictated by their geometrical properties - a one-dimensional shape used in combination with the linear liquid crystal structures described later on - in order to obtain orientation of the rods; their quantum properties - they behave as single photon emitters ${ }^{[7]}$-andtheir polarized emission ${ }^{[6]}$ in order to control the polarization of the emission of single emitters.

(a)
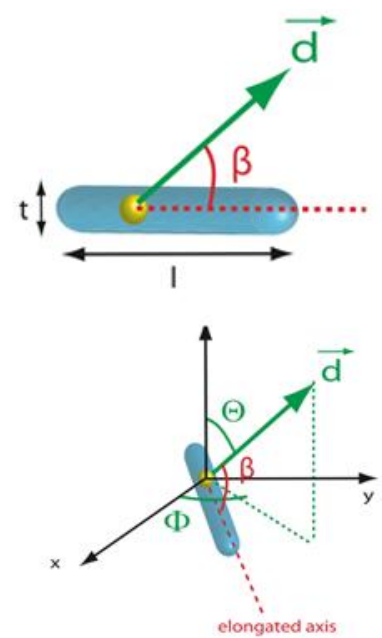

Sample
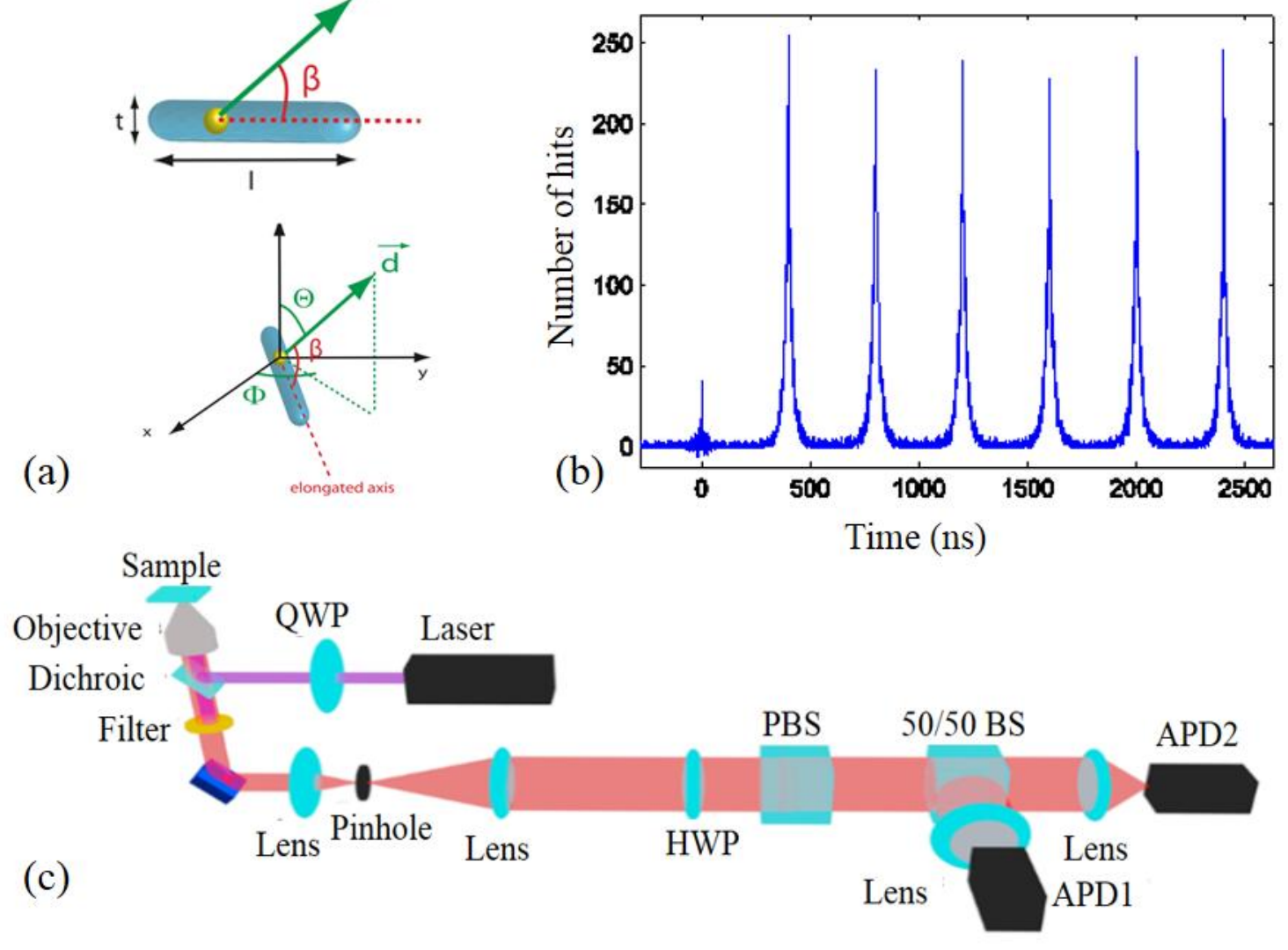

Figure 1. a)Schematic of the DRs with the angle $\beta$ between the DR axis and the dipole indicated, together with the out-of-plane tilt of the dipole, $\theta$, and the in-plane tilt, $\Phi$. b) autocorrelation function for 
a typical DR in $8 \mathrm{CB}$, with $8 \mathrm{CB}$ background contribution (see in Supporting Information S1)substracted following. ${ }^{[50]} \mathrm{c}$ ) Experimental setup during the polarization measurement.

Colloidal core/shell CdSe/CdSDRs were synthesized by using a seeded growth approach. ${ }^{[18,51]}$ They are constituted of a CdSe core with a diameter of $2.9 \mathrm{~nm}$ and surrounded by a rod-like CdS shell (Figure1 a). As confirmed by transmission electron microscopy (TEM) analysis, they present a rod-like shape with an average length $1=23 \mathrm{~nm} \pm 3 \mathrm{~nm}$ and a total thickness $\mathrm{t} \sim 7 \mathrm{~nm}$ (figure $\mathrm{S} 2$ in Supporting Information).The relatively large thickness is responsible for an increased photo-stability and blinking suppression with respect to thin shell DRs, grown with standard techniques. ${ }^{[7]}$ They are surrounded by organic ligands, namelyTOP (trioctylphosphine) and ODPA (octadecylphosphonic acid).

Solid surfaces consisting of a glass substrate with spin-coated and rubbed polyvinyl alcohol polymer (PVA) of thickness 10nm were used as substrate to realize films of a smectic liquid crystal composed of elongated molecules of 4-n-octyl-4'-cyanobiphenyl (8CB). Drops $(40 \mu \mathrm{L})$ of a $8 \mathrm{CB}$ solution in toluene $\left(2.10^{-1} \mathrm{M}\right)$,containing DRs $\left(10^{-13} \mathrm{~mol}\right)$, were spin-coated on top of rubbed PVA-coated substrates at 4,000 rpm during 30 seconds with an acceleration varying between 200 and $300 \mathrm{rpm} . \mathrm{s}^{-1}$. This results in $8 \mathrm{CB}$ films of thickness varying between 100 and $200 \mathrm{~nm}$, where arrays of parallel stripes were visualized by optical microscopy (Figure 2 a).

On PVA rubbed surfaces, a planar anchoring of the liquid crystalline molecules is induced at the interface with the PVA, while a perpendicular (homeotropic) alignment is produced at the air interface. Such an anchoring antagonism leads to distortions of the smecticfilms (Figure $2 b$ and $2 c$ ), commonly named smectic "oily streaks" for film thicknesses in the range $100 \sim 300 \mathrm{~nm} \cdot{ }^{[52-54]}$ The smectic layersare curved between the air and PVA interfaces, stacking on top of each other to form flattened hemicylinders of axis parallel to the substrate and perpendicular to the anchoring orientation, the latter being defined by the PVA rubbing direction (Figure $2 \mathrm{a}$ and $2 \mathrm{~b}$ ). The corresponding hemicylinders' 
periodicity and orientation can be determined by optical microscopy since they are associated with straight linear stripes in the pictures (Figure 2 a). Oily streaks are associated with several highly distorted areas underlined in red in Figure 2 b: -1- the curvature walls, W, between neighboring hemicylinders. ${ }^{[52]}-2$ - the area around the rotation axis $\mathrm{C}$ (Figure $2 \mathrm{~b}$ and $\mathrm{e}$ ). This area has been shown on $\mathrm{MoS}_{2}$ crystalline substrates ${ }^{[5]}$ and rubbed PVA polymer ${ }^{[54]}$ to be formed by a rotating grain boundary, to remove the most curved smectic layers. -3 - the region connecting the flattened hemicylinders to the substrate with a straight grain boundary. ${ }^{[54]}$ These grain boundaries connecting different number of smectic layers from each side, they may be composed of a number of straight dislocations parallel to the hemicylinder axis. ${ }^{[54-56]}$ Smectic dislocations are linear topological defects,made of elastically deformed smectic layers around a linear core defect with a diameter of the order of the smectic layer width, i.e. $3 \mathrm{~nm}$ for $8 \mathrm{CB} .{ }^{[57]}$ The linear cores are expected to behave as efficient traps for nanoparticles. It has been shownthat gold nanoparticles and quantum dotscan be trapped in oily streaks, leading to the formation of straight chains of nanoparticles, parallel to the dislocation. ${ }^{[58]}$

A confocal fluorescence microscopy setup (Figure $1 \mathrm{c}$ )was used with either a mercury lamp source or a laserdiode source to excite single DRs. The emitted light was collected by avalanche photodiodes or a CCD camera. To first observe the liquid crystal on the substrate as well as to locate the DRs, amercury lamp with a filter selecting the $436 \mathrm{~nm}$ linewas used to illuminate the sample. The light reflected at the interface between the liquid crystal and the glass substrate confirms the presence of the oily streaks (Figure 2 a). To locate the DRs themselves, we used a high-pass filter with a $570 \mathrm{~nm}$ cut-off in the detection path, eliminating the reflection from the liquid crystal while allowing the photoluminescence signal from the DRs to get through (wavelength between 575 and $650 \mathrm{~nm}$ ). 


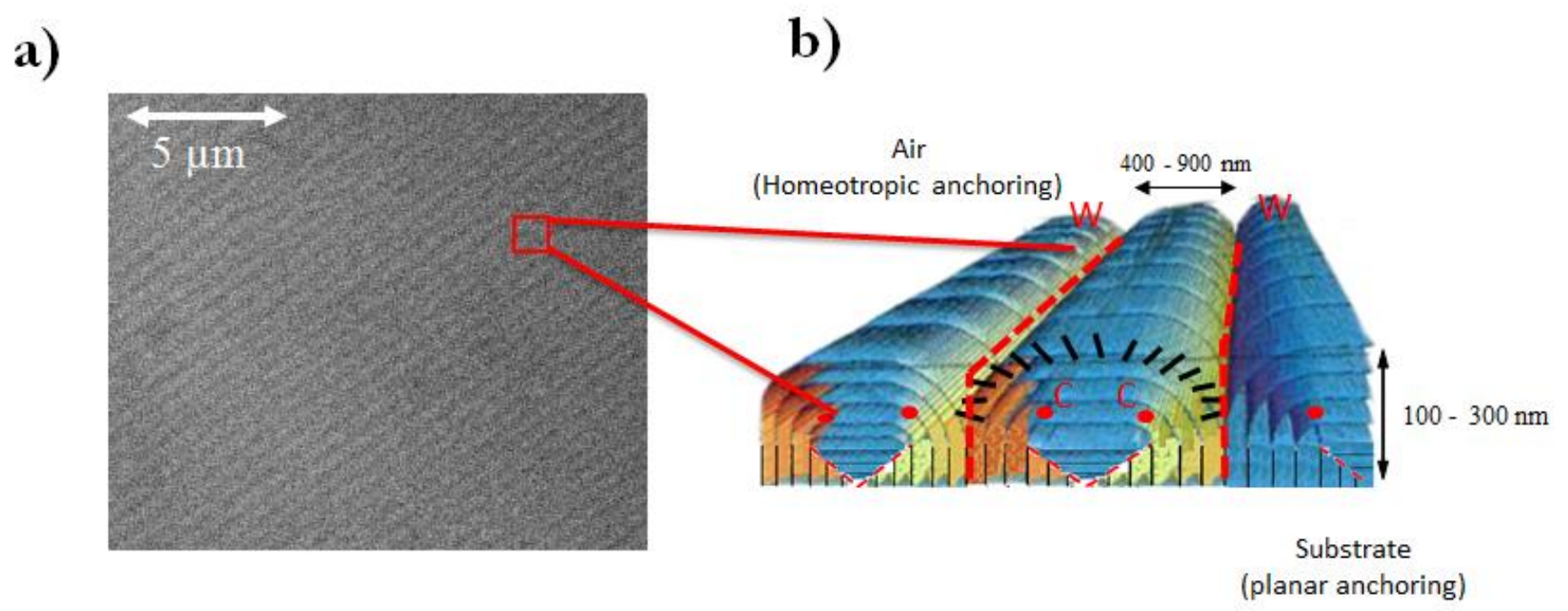

Figure 2. a) Oily streaks observed by optical microscopy on a sample $120 \mathrm{~nm}$ thick, viewed from the top. b) Oily streaks structure with the smectic layers schematically shown in 3D. The centers of curvature, $\mathrm{C}$, and the walls between the hemicylinders, $\mathrm{W}$, and the grain boundary allowing for the connection of the hemicylinders with the substrate, are highlighted in red. e) $A$ zoom on the oily streaks is shown in side view for films of thickness around $150 \mathrm{~nm},{ }^{[54]}$ with the areas withsmectic dislocations parallel to the OX axis, being highlighted with the red crosses.

Acircularly polarized pico-second pulsed laser diode $(\lambda=404 \mathrm{~nm}$, repetition rate $2.5 \mathrm{MHz})$ was then focused on each single DRembedded in the liquid crystal (average optical index 1.57) through a high numerical aperture air objective ( $\mathrm{NA}=0.95)$. As light absorption efficiency of a DR depends on the polarization of the excitation light ${ }^{[39,59]}$, the chosen circular polarization ensures a comparable excitation for DRs with potentially different orientations, allowing similar emission intensities for all particles. Low excitation power below the DR absorption saturation levelwas used in order to limit multi exciton emission and blinking. ${ }^{[7]}$ In a first step, intensity second-order auto-correlation at zero delay measurements $\left(\mathrm{g}^{2}(0)\right)$ were performed with a Hanbury-Brown and Twiss setup consisting of a 50/50 
beam-splitter, separating the photon flow to two avalanche photodiodes and measuring the coincidence counts. The corresponding antibunchingis shown in Figure1 b, with the8CB background fluorescence independently measured and substracted as explained in Ref. 50 (see Supporting Information S1). The peak at zero delay, although not fully absent (probably due to weak multiexciton emission), is much lower than the other peaks, which indicates that we are observing a single isolated DR. It allowsto establish that all the measured DRs are single photon emitters and select the isolated ones for further measurements. In a second step, a rotating linear polarizer made of a half-wave plate (HWP) and a polarizing beam splitter (PBS) wasplaced before the 50/50 beam splitter (Figure1c).The rotation of the half-wave plate allowed the analysisof the polarized component of the beam emitted by each isolatedDR. 90s-long time traces wereregistered with the half-wave plate rotating at a constant speed of $1^{\circ} \cdot \mathrm{s}^{-1}$.Representative results of this measurement are reported in Figure3c for typical raw data.

By virtue of their peculiar emission diagram ${ }^{[6]}$ and the generally high level of emitted linear polarization, measured both in ensemble and single particle spectroscopy $,{ }^{[5,24,}{ }^{25},{ }^{60},{ }^{61]} \mathrm{DRs}$ are commonly associated with one dimensional linear dipoles. ${ }^{[7,25,50]}$

In spherical coordinates with z-axis referred as the microscope optical axis (axis perpendicular to the substrate), the orientation of a dipole can be marked out by its in-plane $\Phi$ and out-of plane $\theta$ angles (Figure $1 \mathrm{a}$ ). Itsemission intensity as a function of the analysis angle $\alpha$ is expressed as: ${ }^{[62]}$

$$
\mathrm{I}(\alpha)=\mathrm{I}_{\min }+\left(\mathrm{I}_{\max }-\mathrm{I}_{\min }\right) \cos ^{2}(\Phi-\alpha) \quad(1),
$$

where $I_{\max }$ (resp. $I_{\min }$ ) corresponds to the maximum (resp. minimum) intensity when $\alpha$ is varied. They depend on the out-of-plane angle $\theta$, on the numerical aperture of the collecting objective and on the environment of the dipole.The angle aitself is the polarization rotation angle caused by the $\lambda / 2$ plate (rotated by an angle $\alpha / 2$ ). Its origin has been calibrated by measuring the signal received when the 
emitted light is replaced by a known polarized signal. The $0^{\circ}$ has been defined by the maximum of intensity for a polarization parallel to theOx axis of the optical microscopy pictures (see Figure $3 \mathrm{a}-\mathrm{b}$ ). The detected intensity is maximum when $\alpha$ equals to the in-plane angle of the dipole $\Phi \pm \pi$. Thereby, Equation (1) allows the assessment of $\Phi$, giving thorough knowledge of the in-plane orientation of each individual DR dipole.

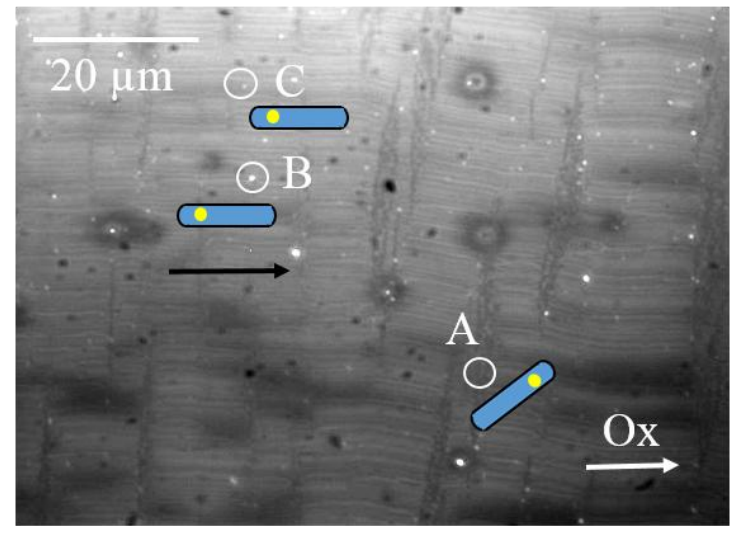

(a)

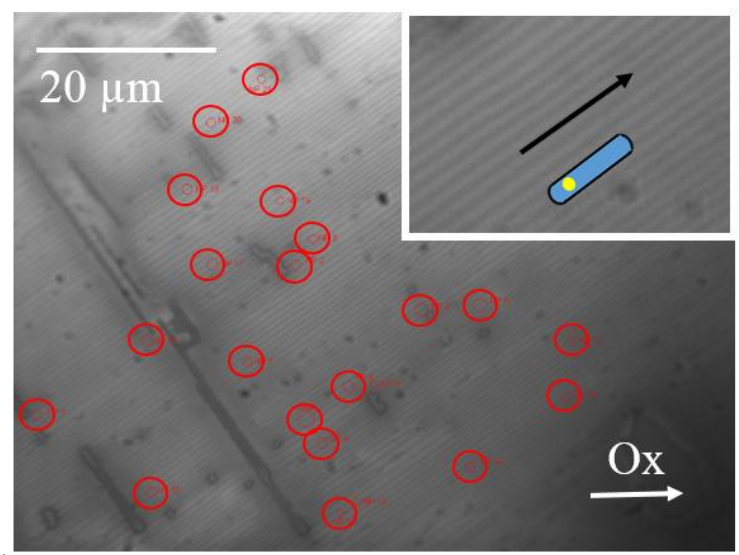

(b)
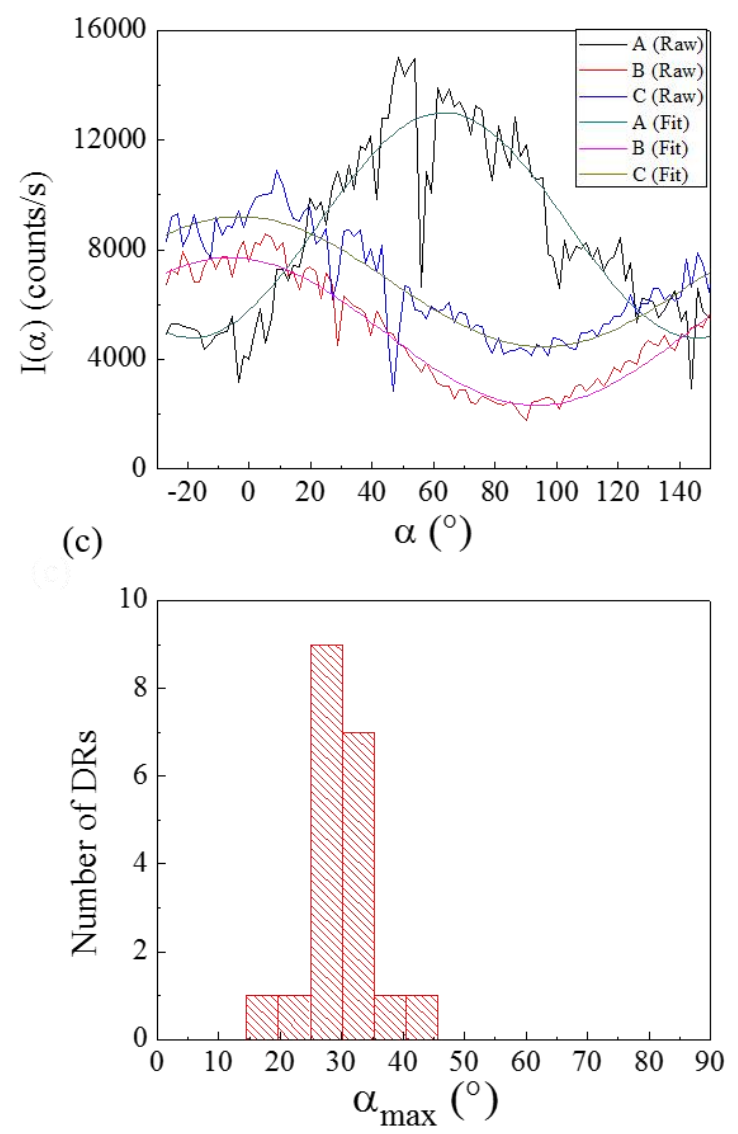

(d)

Figure 3. a) Superposition of the pictures obtained in optical microscopy (oily streaks) and fluorescence microscopy (DRs) on a8CB sample with imperfect PVA polymer rubbing $140 \mathrm{~nm}$ thick. Three specific DRs are chosen for observation. The arrow indicates the axis of the oily streaks, the in-plane orientation of the dipoles (as deduced from Figure 3c) being shown with the blue icon. 
b) $8 \mathrm{CB}$ smectic oily streaks $130 \mathrm{~nm}$ thick observed through optical microscopy with the PVA rubbing performed with a rubbing machine. The position of the DRs is found in the smectic oily streaks, via fluorescence microscopy and is highlighted by red circles on the picture - the inset shows a zoom of size $13.5 \mu \mathrm{m} \times 9 \mu \mathrm{m}$. The arrow indicates the axis of the oily streaks, the in-plane orientation of the dipoles (as deduced from Figure 3d)being shown with the blue icon.

c) Overlay of the photoluminescence intensity emitted by three DRs shown on Figure 3aas a function of the polarization analysis angle $\alpha$. A fit with a $\cos ^{2}(\alpha-\Phi)$ function is superimposed to the three curves

d) Histogram of the angle value corresponding to the fluorescence maximum obtained from the $\cos ^{2}(\alpha-\Phi)$ fit for the 20 DRs measured on Figure 3b

Two different experimental approaches have been used for rubbing the PVA-coated substrates. The firstmethod consisted in rubbing the substrates through a hand-operated procedure, in our case by using cotton sticks, achieving aligned zones, parallel from each other, but extended over small-scale areas only (of the order of several dozens of micrometers - see Figure 3a). These areas are separated by disordered zones, without preferred liquid crystal orientation and thus without oriented oily streaks. The second method uses a rubbing machine providing almost nodisordered zones, essentially showing regular stripes (Figure 3b) aligned along a single direction over the whole sample surface, this direction being perpendicular to the rubbing direction. In Figure 3c, we plot the fluorescence intensity $\mathrm{I}(\alpha)$ emitted by three individual DRs, as a function of the polarization analysis angle $\alpha$, for the manually-rubbed sample covered with a layer approximately $100 \mathrm{~nm}$ thick. For each DR, the measured intensity shows the typical $\cos ^{2}(\alpha-\Phi)$ dependence of eq. (1), as shown from the comparison between raw data and a $\cos ^{2}(\alpha-\Phi)$ fit. If we compare two DRs localized on two oriented areas (DRs B and C on Figure 3a), we obtain a similar phase of $\mathrm{I}(\alpha)$, moreover with an intensity maximum at $\alpha=0^{\circ}$, corresponding to the stripes orientation 
(Figure 3a). If we compare them with one DR on a disordered area (DR A on Figure 3a), different I( $\alpha$ ) phases are obtained. It brings the conclusion that the fluorescencepolarization is maximum in a welldefined directionparallel to the stripes, only when the DRs are localized in oriented oily streaks. The same result isobtained for the $\mathrm{I}(\alpha)$ curves of the DRs of the homogeneously rubbed sample (red circles in Figure 3b). On Figure 3d, the histogram of the angle values corresponding to the fluorescence maximum for the $20 \mathrm{DRs}$ of Figure $3 \mathrm{~b}$ is presented. A common phase value for the different DRs appears clearly, with an average of $\Phi=29.9^{\circ}$ and a standard deviation of $5.6^{\circ}$. This average value is the same than the stripes orientation, visible on optical microscopy (Figure 3b), with a measurement uncertainty of $\pm 1^{\circ}$. If now we consider a larger number of single DRs located in two different samples of similar $8 \mathrm{CB}$ thickness of the order of $100 \mathrm{~nm}$ (52 DRs), we find again a well-defined value for the fluorescence anisotropy, corresponding to the stripes orientation, with a standard deviation of $8^{\circ}$. This demonstrates that, not only the DRsfluorescence share a common orientation, but also that this orientation is the one of the oily streaks defects.

The hypothesis of an influence of the liquid crystal birefringence on the fluorescence polarization anisotropy of the DRs can be ruled out for two reasons. First it has been shown recently that theexcitation anisotropy of DRs is mainly affected by the nature of the ligandsdirectly around the DRs. ${ }^{[25]}$ Second,several ensemble measurements of polarization anisotropy have been conducted within nematic liquid crystals. ${ }^{[22,46,48-49,63]}$ This led to a maximum fluorescence parallel to the nematic director (extraordinary index) in contrast with the present case.In smecticoily streaks, Figure 3 shows that the fluorescence is maximum for a polarization along the stripes, in other words perpendicular to the smectic direction and corresponding to the ordinary index of the $8 \mathrm{CB}$. The fluorescence is minimum for a polarization perpendicular to the stripes, corresponding to a combination between ordinary and extraordinary index.For bothnematicand smecticliquid crystals, the fluorescence anisotropy canbe 
attributed to an in-plane orientation of the DRdipole, imposed by the liquid crystal and not to an optical birefringence effect. In nematic liquid crystals the fluorescence polarization anisotropyis related to an orientation of the nanorodsparallel to the liquid crystal director, as it is most often the case due to the liquid crystal anchoring at the nanorods surface. ${ }^{[22,39-40,46,48-49,63]}$ In smectic oily streaks, the in-plane projection of the DRs dipole is oriented parallel to the oily streaks stripes (scheme of the dipoles on Figure 3). It is thus parallel to the linear defects, and, perpendicular to the liquid crystal director (Figure 2). This suggests a different physical mechanism for the aligning phenomenon in smectic oily streaks compared to orientednematic liquid crystals.

The dipole orientation with respect to the smectic texture is confirmed bya defocused microscopy technique, ${ }^{[6,48]}$ already used toassessDRs dipole orientation. ${ }^{[6,59]}$ It has been employed by means of an oil immersion microscope objectivewith high numerical aperture (NA1.4)positioned $500 \mathrm{~nm}$ away from the focal point. As shown in Figure 4, bright and dark arcsappear around each original focused spot, confirming the dipolar nature of theemission. ${ }^{[6,49,59,62,64-65]}$ These defocusing patterns giveinformation on both in-plane and out-of-plane dipole orientation, ${ }^{[62,}{ }^{64-65]}$ if it is taken into account that they are influenced by several other parameters such as NA of the objective, dielectric environment (liquid crystal and air interface) and that they are significantly dependent on the distance between the objective focal planeand the sample $\mathrm{e}^{[49,51-52]}$ For linear dipoles, defocused images displaying lobe patterns with a single symmetry axis correspond to dipoles neither horizontal nor vertical, but rather tilted with respect to the substrate.By connecting the two minima of the internal arcs,the dipole in-plane component orientation can be assessed:as shown in the upper-left inset of Figure 4, it is parallel to the oily streaks axis, highlighted in the bottom-right inset.Thisconfirms the result gained from Figure 3. Importantly, again, this specific orientation is common to the majority of the isolated DRswithin the rubbed area (See Figure S3). 


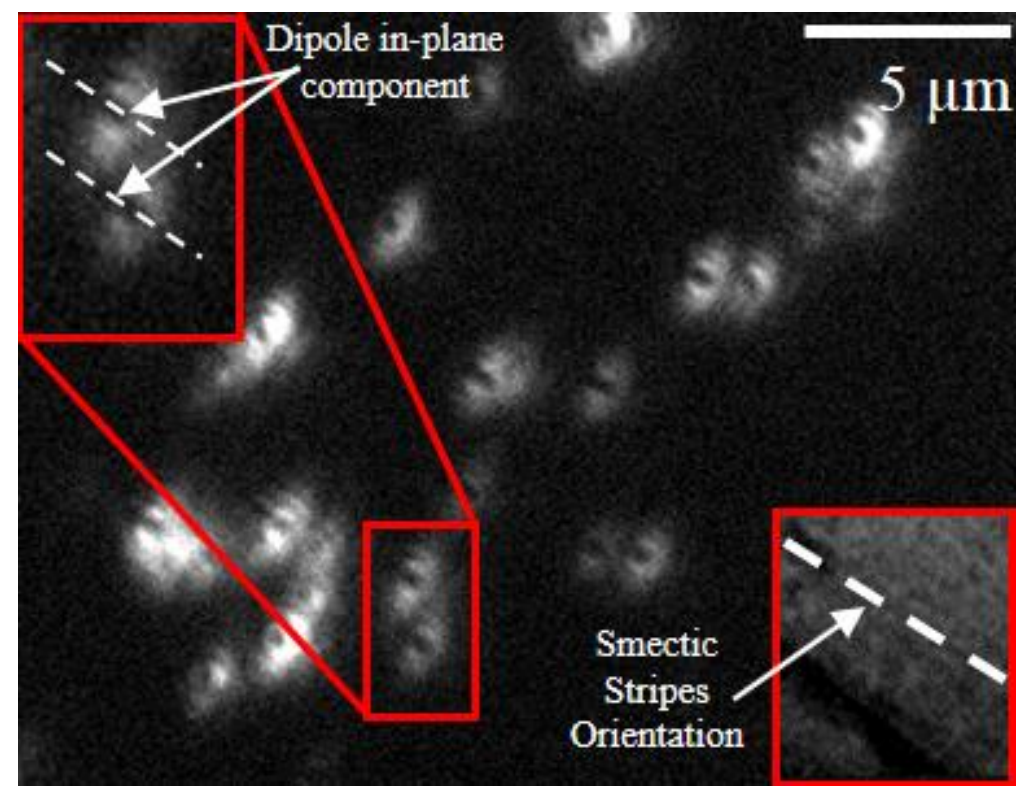

Figure 4. (Main) Photoluminescence of DRs illuminated with the $436 \mathrm{~nm}$ band of a $\mathrm{Hg}$ lamp in defocused configuration:the objective is defocused by setting the focal plane $500 \mathrm{~nm}$ far from the DR; the image is takenafter filtering out the reflection with a high-pass filter to keep the photoluminescence. (Inset-left)Zoom on two defocusing spots. (Inset-right; $5 \times 6 \mu \mathrm{m}$ ) In absence of high-pass filter and in focused configuration, the oily streaks are observed with the same source.

On Figure 4, the asymmetry of the defocusing spots shows a tilt of the DRs dipoles out of the substrate plane. In order to supplement these defocusing measurements, we have considered the degree of linear polarization $\delta$ for the emitted light of the single DRsin the liquid crystal matrix: $\delta=\left(\mathrm{I}_{\max ^{-}}\right.$ $\left.I_{\min }\right) /\left(I_{\max }+I_{\min }\right) . I_{\max }$ and $I_{\min }$ are obtained from polarization analysis curves as in Figure $3 c$, after careful backgroundsubstraction. For DRsapproximated by linear dipoles, ${ }^{[7,28,59]} \delta$ can be theoretically calculated taking into account the dielectric nature of the environment of the dipole. ${ }^{[62]}$ The theoretical curve of the degree of polarization $\delta$ as a function of the dipole out-of plane tilt, $\theta$, with respect to the microscope optical axis (normal to the substrate), is shown on Figure 5a for a linear dipole located in a 1.5 index 
medium, whose emission is collected by a $0.95 \mathrm{NA}$ air objective ${ }^{[62]} \delta \approx 1$ for $\theta=90^{\circ}$, which is a horizontal dipoleand $\delta=0$ for $\theta=0^{\circ}$, which is a vertical dipole.

We measured the degrees of polarization of 52DRs dispersed in two similar samples of liquid crystal thickness around $100 \mathrm{~nm}$, including the one of Figure $3 \mathrm{~b}$. We removed, for this measurement, the signal of theDRsnot aligned within $6^{\circ}$ to the liquid crystal stripesandmost probably corresponding to nanoparticles of different shapes (see TEM image displayed in figure S2) ${ }^{[25]}$ The obtained histogram of degree of polarization,for the 42 remaining DRs, is shown on Figure 5b. A well-defined peak is evidenced between 0.6 and 0.7 . As shown by Figure $5 \mathrm{a}$, this limited value of polarization is consistent with theout-of plane tilt of the emitter dipolesevidenced on the defocalization results of Figure 4. It may also be associated with DRsnot fully polarized, as shown in a number of ensemble measurements, ${ }^{[5,24-25]}$ in particularforCdSe/CdSDRs of similar ratio core diameter over DR diameter, the role of this last parameter being recently underlined ${ }^{[46]}$ The broad distribution of degrees of polarization observed on Figure $5 \mathrm{~b}$ may be related to the dispersion of size and shape of the DRs (see figure S2), to the distribution of core shape, ${ }^{[24-25,60-61]}$ together with possible distribution of dipole out-of plane orientation.
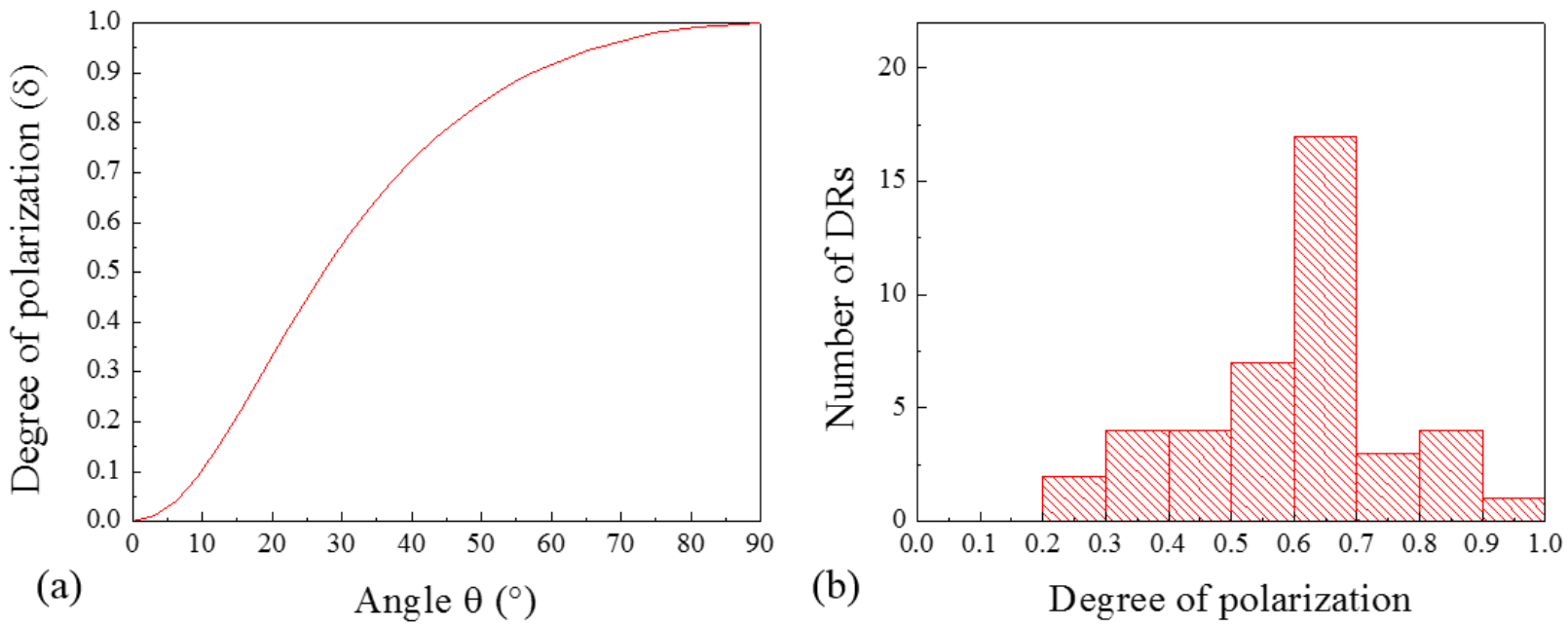
Figure 5. (a) Degree of polarization of a linear dipole located in a 1.50 refractive index medium, whose transmitted emission is collected by a 0.95 NA air objective, as a function of its out-of-plane angle $\theta$. This curve does not depend on the distance to the interface; (b) Histogram of the measured degrees of polarization for 42DRs in 8CB liquid crystal films of thickness $100 \mathrm{~nm}$.

In a similar geometry of single polarization measurements performed on the same kinds of DRs deposited on glass, with, in addition, an AFM mounted on an inverted microscope to probe the single DRs orientation, it has been recentlyevidenced that the maximum of fluorescence was obtained for a polarization parallel to the single DRsaxis. ${ }^{[2]}$ Together with our observation of in-plane dipoles parallel to the oily streaks stripes visible by optical microscopy, this suggests thattheDRsaxis is itselforiented parallel to the stripes.DRs are thus perpendicular to the $8 \mathrm{CB}$ anchoring direction, but parallel to the $8 \mathrm{CB}$ hemicylinder axis and thus parallel to the expected dislocations of oily streaks. the evidence of nanorod trapping, allowing for nanorods orientation as well, may confirm the presence of aligned dislocations in the oily streaks structure. Smectic dislocations are characterized by a disordered linear core of high energy with a diameter of the order of a fewnanometers. ${ }^{[57]}$ The DRs width (with a total of $7 \mathrm{~nm}$ ) matches the one of the smectic dislocation cores. In addition to their elongated shape, this size matching promotes their trapping inside the line defects, in agreement with the previously evidenced phenomenon of nanospheres trapping by topological LC defects, ${ }^{[66-69]}$. The induced orientation of the DRs, parallel to the dislocation cores (Figure 3), may correspond to a precise localization of the DRs within the disordered cores. This localization of DRswithin and parallel to the dislocation coreindeed maximizes the volume of disordered liquid crystal expelled by the DRs and thus decreases the disorder energy of the smectic liquid crystal film in the presence of dislocations. If we consider elementary smecticdislocations, their energy per unit of length has been measured in free standing smectic filmsto be $0.5 \mathrm{kT} . \AA^{-1} \cdot{ }^{[70]}$ Asingle DR of length $1=23 \mathrm{~nm}$, trapped in the dislocation core and parallel to its axis, 
allows todecrease the liquid crystal disorder energy by $115 \mathrm{kT}$, leading to a significant advantage for the liquid crystal film. TheDRs, once trapped in smecticdislocations, may be particularlywell-stabilized. Only motion of the DRs along the dislocations cores may occur, in agreement with experimental observations. Our results may consequently correspond toDRs, trapped within the liquid crystal dislocations, parallel to the substrate and parallel to the $8 \mathrm{CB}$ linear stripes.

The origin of this nanorod induced orientation is obviously different from the one induced in nematics, this latter being driven by the anchoring of liquid crystal molecules at the DRsurface, usually leading to DRsoriented parallel to the nematic director. ${ }^{[39-40]}$ In contrast, in smectic oily streaks, the induced orientation is parallel to the dislocations, thus perpendicular to the liquid crystal director. Generally speaking, if the nanorod diameter is small enough to avoid, outside the dislocations core, a newsmectic distortion/disorder energy larger than the energy advantage associated with the nanorod presence in the dislocation core, we expect no liquid crystal-induced aggregation. As a consequence, contrary to the nematic case, the liquid crystal anchoring at the DR surface is also expected to have almost no influence and the DRsmay be efficiently oriented by the dislocation, independently of the liquid crystal anchoring at their surface. This appears in agreement with the fact that nanospheres surrounded by different ligands, alkylthiols, aresimilarly linearly oriented, since they formchains parallel to the dislocations in the $8 \mathrm{CB}$ oily streaks. ${ }^{[58]}$

Ifthe DR axis isparallel to the smectic dislocations, an out-of plane tilt of the DR dipole corresponds to an emitting dipole tilted by an angle $\beta$ (see Figure 1a) from the horizontal nanorodaxis, in agreement with ref [59]. In presence of a $\beta$ angle between the fixed DRaxis and the dipolewe anticipatea free rotation of the dipole around the $\mathrm{DR}^{[59]}$. In contrast, Figure 3 and 4 suggest that the dipoles remain all oriented in the plane defined by the rod and the normal to the substrate. The origin of such a strictly 
induced out-of-plane dipole orientation is not clear. Surface charges may exist on these DRs, related either to the crystallographic orientation of the nanorodsurface ${ }^{[71]}$ and/or to dangling bonds unsaturated by the surface ligands. ${ }^{[72]} \mathrm{A}$ symmetry breaking in the smectic phase at the DR interfacemay occur, due to the charges at the DR surface, which may orient all neighbouring $8 \mathrm{CB}$ molecular dipoles. ${ }^{[71-72]}$ Strong electric dipolar interactions between the $8 \mathrm{CB}$ molecular dipoles $(4.9 \mathrm{D})$ and the DR dipoles ${ }^{[71]}$ maythus favour DR dipole orientationin the plane parallel to the $8 \mathrm{CB}$ stripes and perpendicular to the substrate. This outstanding property of the smectic oily streaks evidences a good control of single emitters since, with our smectic liquid crystal/DRs composites, we create a dispersion of single photons emitters of controlled polarization phase, oriented along a unique direction for one given sample. It opens the way to macroscopic nanoemitter lines, sources of well polarized single-photon emission.Generally speaking, the ability to orient nanorodsshows a high potential of thesmectic oily streaks for the control of nanoparticles'optical properties. This goes well beyond semi-conducting nanoparticles, being, for example, well adaptedfor inducing anisotropicplasmonic extinction of metallic nanorods.

In conclusion, we showed that the oily streak structure created by a liquid crystal deposited on a rubbed polymer induces an alignment along a unique direction of the dot-in-rods (DRs) diluted in the liquid crystal matrix,leading to fixed orientation for the emitting in-plane dipoles. This has been shown at the single particle levelthanks to the evidence of the intensity second-order autocorrelation function of each DR.Over a collection of DRs, we found the fluorescence polarization orientations to be along the liquid crystal stripes within a $8^{\circ}$ standard deviation. The dipoles of the DRs used in this study thus behave as individual polarized single photon emitters. This orientation along the oily streaks axis is likely due to the trapping of the DRs within the smectic dislocation cores, oriented along a single direction, together with electric dipole-dipole interactions between the DRsdipoles and the liquid crystal molecules. This leads to an orientation of the DRs without aggregation, a priori independently of the 
ligand's nature around the DRs. This work thus shows the feasibility of a large-scale orientation of anisotropic emitters without the stringent requirements shown in previous studies (control of the ligand's nature, continuity of the sample, particles in direct contact with each other or under the influence of an external electric field). The experiment demonstrates the trapping efficiency of smecticdislocations which not only trap but also align elongated nano-objects, along a single direction for a given sample, thanks to the use of rubbed polymer substrates. These results open the way for not only orienting dot-inrods but also for creating chains of oriented dot-in-rods emitting polarized light.

\section{Acknowledgements}

The authors would like to thank Dominique Demaille and Francis Breton.For financial support, they also would like to thank the AgenceNationale de la Recherche (P3N Delight, JCJC Ponimi and PNanoNanodiellipso), the programsC'nanoNanoPlasmAA and C'Nano Sophopol, the PUF program of the French Embassy in the United States of America.

Supporting information

S1: Presentation of the treatment of the background data during the antibunching measurement.S2:

Pictures of the CdSe:CdS dot-in-rod particles taken with a Transmission Electron Microscope; S3:

Defocused image of aligned dipoles over a large area $(60$ by $80 \mu \mathrm{m})$ area; S4: Absorption and photoluminescence emission spectra for the CdSe:CdS dot-in-rod particles used for the work presented in this paper. Supporting Information is available from the Wiley Online Library or from the author.

S1: Presentation of the treatment of the background data during the antibunching measurement. 
In order to take into account the contribution from the background, we used the procedure described in the appendix of ${ }^{[50]}$. This procedure has already demonstrated its ability to describe experimental data with great accuracy (see fig. 2 of [50]). Let us summarize this procedure used here for the 8CB-nanorod system, keeping the notations of Ref. [50].

(a) We measure independently the fluorescence of a portion of $8 \mathrm{CB}$ without nanorod and we measure the 8CB fluorescence decay rate: Gamma $\_$p $=0.12 \mathrm{~ns}^{-1}$

(b) From the file used to plot the nanorodantibunching curve, which contains the arrival time of each photon individual, we can plot the detected intensity as a function of time. The detected intensity is a sum of the respective intensities of the fluctuating nanorod emission (I_NC(t)), the 8CB emission (B_P), and the photodiode dark counts (B_0). Because of some residual blinking, this curve shows some brief time intervals when the nanorod is not emitting: the detected intensity is then the background intensity B_0 + B_P $=2000$ counts per second (cps). By substracting this background contribution, we can calculate the average nanorod intensity $<$ I_NC(t) $>=14.6 \mathrm{kcps}$ and the average squared intensity $<$ I_NC ${ }^{2}(t)>=4.310^{8} \mathrm{cps}^{2}$.

(c) Finally, from the same measured file, it is possible to plot the decay curve, which has a constant component (the dark counts, which are not correlated to the excitation laser pulses) and a decay component (sum of the nanorod and $8 \mathrm{CB}$ decays, which can be distinguished as the nanorod decay is much slower than the $8 \mathrm{CB}$ decay). The constant component of the decay curve yields the dark counts rate: B_0 $=410$ cps. The slow decay component yields the nanorod decay rate: Gamma $=0.046 \mathrm{~ns}^{-1}$.

We have measured all the experimental constants necessary to describe the antibunching curve following the model of ref. [50]. The antibunching curve is an intensity autocorrelation curve, thus a 
sum of the background and nanorod autocorrelations and the background-nanorod correlation. On Fig. 1, in order to plot only the nanorod autocorrelation, we plot the experimental antibunching curve from which we substract the theoretical background autocorrelation (Eq. 19 of ref. [50]) and background nanorod correlation (eq. 20 of ref. [50]).

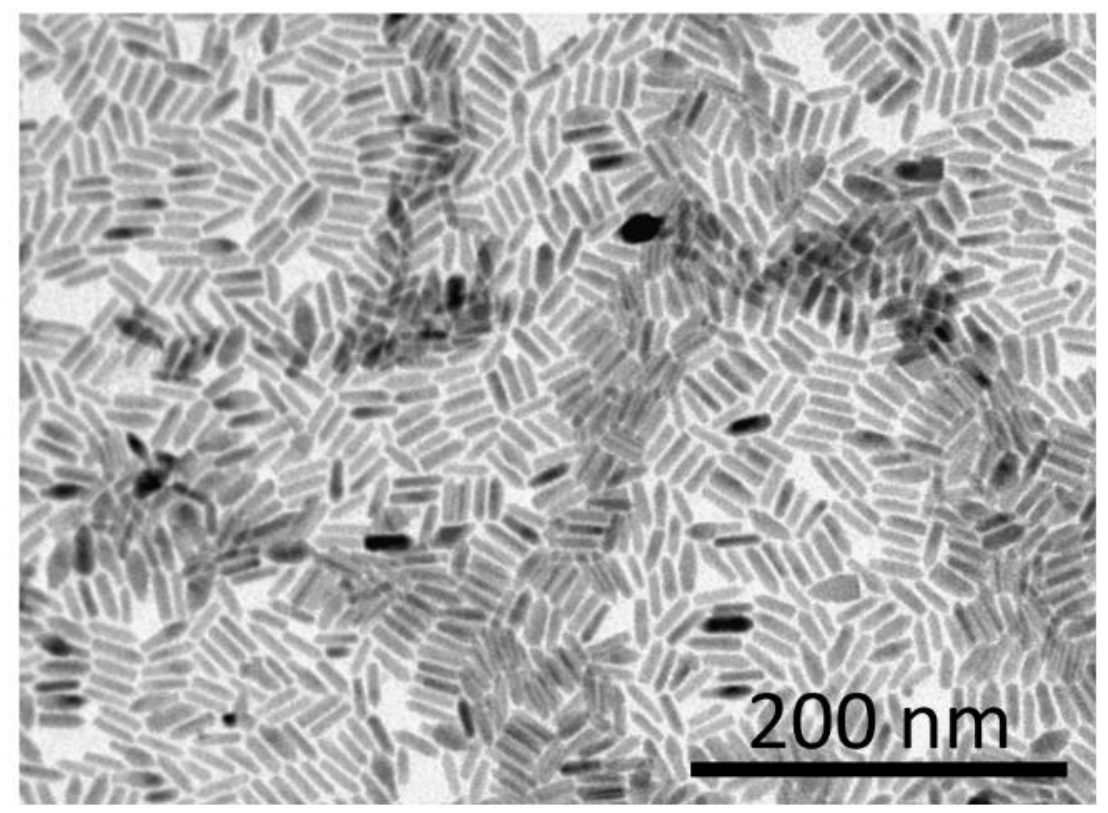

S2: Pictures of the CdSe:CdS dot-in-rod particles taken with a Transmission Electron Microscope. 


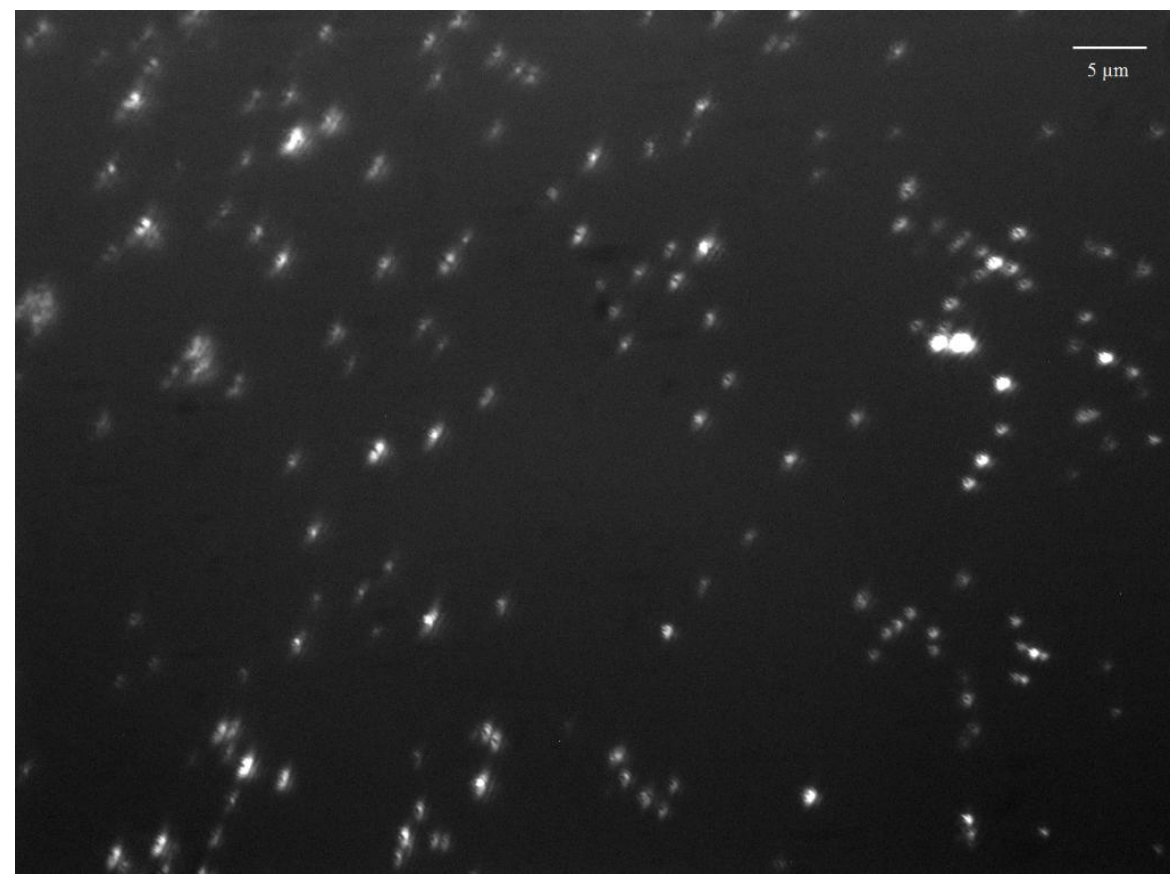

S3: Defocused image of aligned dipoles over a large area (60 by $80 \mu \mathrm{m})$ area

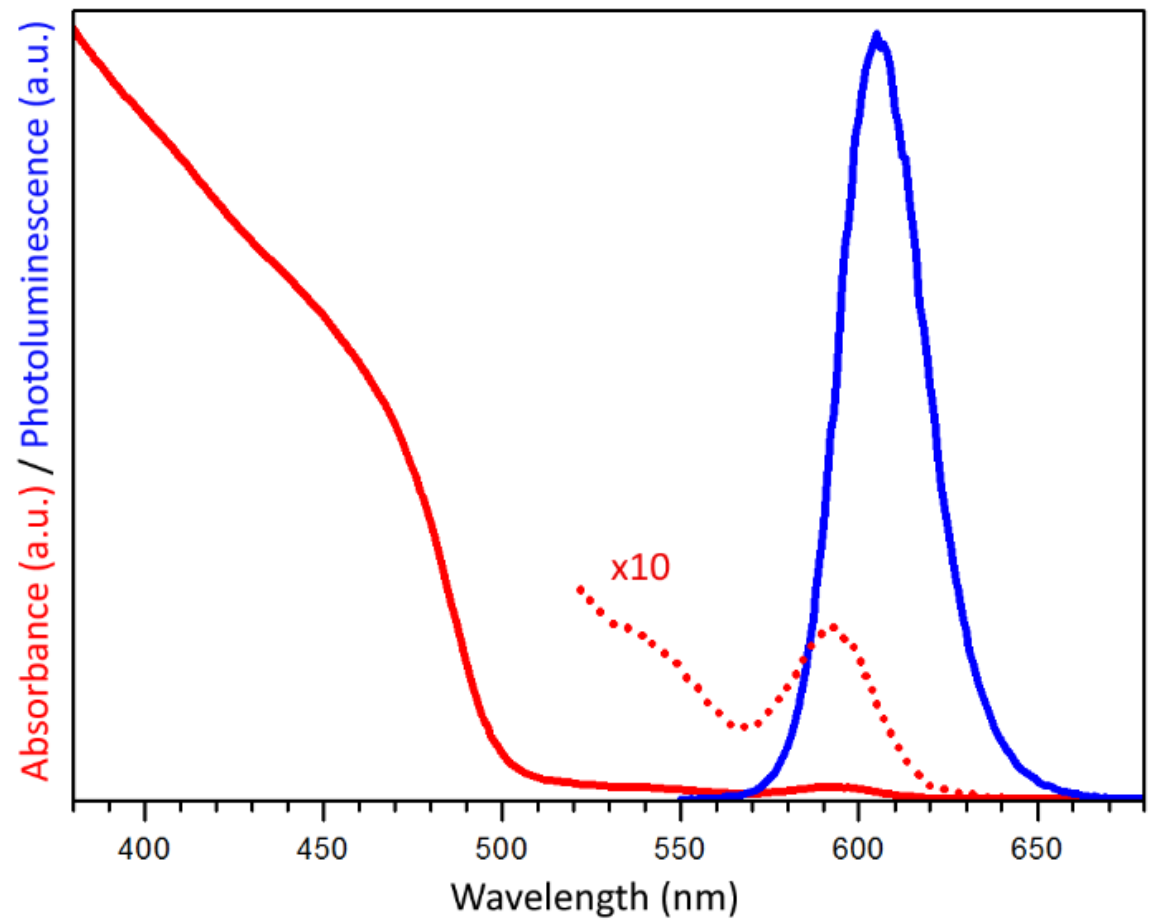

S4: Absorption and photoluminescence emission spectra for the CdSe:CdS dot-in-rod particles used for the work presented in this paper. 
References:

[1] M. D. Eisaman, J. Fan, A. Migdall and S. V. Polyakov, Rev. Sci. Instrum.,2011, 82, 071101.

[2] A. Shabaev and A. L. Efros, NanoLett.,2004, 4, 1821.

[3] R. Krahne, G. Morello, A. Figuerola, C. George, S. Deka and L. Manna, Phys. Rep., 2011, 501, 75.

[4] X. Chen, A. Nazzal, D. Goorskey, M. Xiao, Z. A. Peng, and X. G. Peng, Phys. Rev. B, 2001, 64, 245304.

[5] D. V. Talapin, R. Koeppe, S. Gotzinger, A. Kornowski, J. M. Lupton, A. L. Rogach, O. Benson, J. Feldmann and H. Weller, NanoLett.,2003, 3, 1677.

[6] F. Pisanello, L. Martiradonna, G. Leménager, P. Spinicelli, A. Fiore, L. Manna, J.-P. Hermier, R. Cingolani, E. Giacobino, M. De Vittorio and A. Bramati, Appl. Phys. Lett., 2010, 96, 033101

[7] F. Pisanello, G. Lemenager, L. Martiradonna, L. Carbone, S. Vezzoli, P. Desfonds, P. D. Cozzoli, J. P. Hermier, E. Giacobino, R. Cingolani, M. De Vittorio and A. A. Bramati, Adv. Mat., 2013, 25, 1974.

[8] A. Qualtieri, F. Pisanello, M. Grande, T. Stomeo, L. Martiradonna, G. Epifani, A. Fiore, A. Passaseo and M. De Vittorio, Microel. Eng., 2010, 87, 1435.

[9] F. Pisanello, A. Qualtieri, T. Stomeo, L. Martiradonna, R. Cingolani, A. Bramati and M. De Vittorio, Opt. Lett.,2010, 35, 1509.

[10] C. H. Bennett, G. Brassard, Proceedings of IEEE International Conference on Computers, Systems and Signal Processing, 1984, 175, 8.

[11] Y. Amit, A. Faust, I. Lieberman, L. Yedidya and U. Banin, Phys. Stat. Sol. A, 2012, 209, 235. 
[12] A. Ghezelbash, B. Koo and B. A. Korgel, NanoLett.,2006, 6, 1832.

[13] L. S. Li and A. P. Alivisatos, Phys. Rev. Lett.,2003, 90, 097402.

[14] M. Artemyev, B. Moller and U. Woggon, NanoLett.,2003, 3, 509.

[15] L. S. Li, J. Walda, L. Manna and A. P. Alivisatos, NanoLett.,2002, 2, 557.

[16] C. Querner, M. D. Fischbein, P. A. Heiney and M. Drndic, Adv. Mat.,2008, 20, 2308.

[17] D. V. Talapin, E. V. Shevchenko, C. B. Murray, A. Kornowski, S. Forster and H. Weller, JACS, 2004, 126, 12984.

[18] L. Carbone, C. Nobile, M. De Giorgi, F. D. Sala, G. Morello, P. Pompa, M. Hytch, E. Snoeck, A. Fiore, I. R. Franchini, M. Nadasan, A. F. Silvestre, L. Chiodo, S. Kudera, R. Cingolani, R. Krahne and L. Manna, NanoLett.,2007, 7, 2942.

[19] A. Rizzo, C. Nobile, M. Mazzeo, M. De Giorgi, A. Fiore, L. Carbone, R. Cingolani, L. Manna and G. Gigli, ACS Nano, 2009, 3, 1506.

[20] P. Yang and F. Kim, ChemPhysChem, 2002, 3, 503.

[21] S. Gupta, Q. L. Zhang, T. Emrick and T. P. Russell, NanoLett.,2006, 6, 2066.

[22] K. J. Wu, K. C. Chu, C. Y. Chao, Y. F. Chen, C. W. Lai, C. C. Kang, C. Y. Chen and P. T. Chou, NanoLett.,2007, 7, 1908.

[23] Z.Hu, M. D. Fischbein, C. Querner and M. Drndic, NanoLett.,2006, 6, 2585.

[24] I. Hadar, G. B. Hitin, A. Sitt, A. Faust and U. Banin, J. Phys ChemLett, 2013, 4, 502. 
[25] B. T. Diroll, T. Dadosh, A. Koschitzky, Y. Goldman and C. B. Murray, J Phys Chem C, 2013, 117, 23928.

[26] C. Knorowski and A. Travesset, EPL, 2012, 100, 56004.

[27] K. Thorkelsson, J. H. Nelson, A. P. Alivisatos and T. Xu, NanoLett.,2013, 13, 4908.

[28] R. A. M. Hikmet, P. T. K. Chin, D. V. Talapin, H. Weller, Adv. Mat.2005, 17, 1436.

[29] F. A. Aldaye, A. L. Palmer and H. F. Sleiman, Science, 2008, 321, 1795.

[30] V. Georgakilas, D. Gournis, V. Tzitzios, L. Pasquato, D. M. Guldi and M. Prato, J. Mater. Chem., 2007, 17, 2679.

[31] H. Qi and T. Hegmann, J. Mater. Chem., 2008, 18, 3288-3294

[32] O. Stamatoiu, J. Mirzaei, X. Feng and T. Hegmann, Top. Curr. Chem., 2012, 318, 331-393. 1-8

[33] H. K. Bisoyi, S. Kumar, Chem. Soc. Rev., 2011, 40, 306.

[34] J. P. F. Lagerwall and G. Scalia, Curr. Appl. Phys., 2012, 12, 1387.

[35] J. Mirzaei, M. Reznikov, T. Hegmann, J. Mater. Chem., 2012, 22, 22350.

[36] C. Blanc, D. Coursault and E. Lacaze, Liq. Crys. Rev., 2013, 1, 83.

[37] J. M. Winkler, S. G. Lukishova and L. J. Bissell, Journal of Physics: Conference Series 414, 2013, 012006

[38] S. G. Lukishova, A. W. Schmid, R. Knox, P. Freivald, L. J. Bissell, R. W. Boyd, C. R. Stroud and K. L. Marshall, J. Mod. Opt.,2007, 54, 417. 
[39] S. V. Burylov and Y. L. Raikher, Phys Lett A., 1990, 149, 279.

[40] S. V. Burylov, Y. L. Raikher, Phys. Rev. E., 1994, 50, 358.

[41] Q. Liu,Y. Cui,D. Gardner,X. Li,S. Heand I. I. Smalyukh, NanoLett.2010, 10, 1347.

[42] S. Umadevi, F. Xiang and T. Hegmann, Adv. Func. Mat.,2013, 23, 1393.

[43] O. Buluy, S. Nepijko, V. Reshetnyak, E. Ouskova, V. Zadorozhnii, A. Leonhardt, M. Ritschel, G. Schönhense and Y. Reznikov, Soft. Mat.,2011, 7, 644.

[44] M. R. Thomas, S. Klein, R. J. Greasty, S. Mann, A. W. Perriman, R. M. Richardson, Adv. Mater. 2012, 24, 4424 .

[45] J. S. Pendery, O. Merchiers, D. Coursault, J. Grand, H. Ayeb, R. Greget, B. Donnio, J.-L. Gallani, C. Rosenblatt, N. Félidj, Y. Borensztein and E. Lacaze, Soft Matter, 2013, 9, 9366.

[46] M. V. Mukhina, V. V. Danilov, A. O. Orlova, M. V. Fedorov, M. V. Artemyev and A. V. Baranov, Nanotechnology, 2012, 23, 325201.

[47] Q. Liu, Y. Yuan and I. I. Smalyukh, Nanoletters, 2014, 14, 4071.

[48] H. S. Chen, C. W. Chen, C. H. Wang, F. C. Chu, C. Y. Chao, C. C. Kang, P. T. Chou and Y. F. Chen, J. Phys. Chem. C, 2010, 114, 7995.

[49] A. L. Rodarte, C. G. L. Ferri, C. Gray, L. S. Hirst, and S. Ghosh, Emerging Liquid Crystal Technologies VII, Proc. of SPIE, 2012, 8279, 82790H, doi:10.1117/12.906235

[50] C. Vion, P. Spinicelli, L. Coolen, J. M. Frigerio, J. P. Hermier and A. Maître,Optics Express, 2012, 18,7440 . 
[51] J. T. Hu, L. S. Li, W. D. Yang, L. Manna, L.; L. W. Wang and A. P. Alivisatos, Science, 2001, $292,2060$.

[52] J. P. Michel, E. Lacaze, M. Alba, M. Gailhanou, M. de Boissieu and M. Goldmann, Phys. Rev. E, 2004, 70, 011709.

[53] B. Zappone and E. Lacaze, Phys. Rev. E, 2008, 78, 061704.

[54] D. Coursault, B. H. Ibrahim, L. Pelliser, B. Zappone, A. de Martino, E. Lacaze and B. Gallas, Optics Express, 2014, 22, 23182.

[55] J. P. Michel, E. Lacaze, M. Goldmann, M. Gailhanou, M. de Boissieu and M. Alba, Phys. Rev. Lett.,2006, 96, 027803.

[56] C. Williams and M. Kléman, J. Phys. Coll., 1975, 36, 315.

[57] M. Kleman, Points, lines, and walls: In liquid crystals, magnetic systems, and various ordered media, 1977, Book 1

[58] D. Coursault, J. Grand, B. Zappone, H. Ayeb, G. Levi, N. Felidj and E. Lacaze, Adv. Mat.,2012, $24,1461$.

[59] C. Lethiec, F. Pisanello, L. Carbone, A. Bramati, L. Coolen and A. Maître, submitted to New J. Phys., 2014, arXiv:1401.1030v1 [physics.optics]

[60] B. T. Diroll, A. Koschitzky and C. B. Murray, J. Phys. Chem. Lett., 2014, 5, 85.

[61] A. Sitt, A. Salant, G. Menagen and U. Banin, NanoLett.,2011, 11, 2054. 
[62] C. Lethiec, J. Laverdant, H. Vallon, C. Javaux, B. Dubertret, C. Schwob L. Coolen and A. Maître,Phys. Rev. X, 2013, 4, 021037

[63] V. V. Danilov, M. V. Artem'ev, A. V. Baranov, G. M. Ermolaeva, N. A. Utkina and A. Khrebtov, I., Optics And Spectroscopy, 2008, 105, 37.

[64] M. Böhmer and J. Enderlein, J. Opt. Soc. Am. B, 2003, 20, 554.

[65] D. Patra, I. Gregor and J. Enderlein, J. Phys. Chem. A, 2004, 108, 6836.

[66] H. Yoshida, Y. Tanaka, K. Kawamoto, H. Kubo, T. Tsuda, A. Fujii, S. Kuwabata, H. Kikuchi and M. Ozabi, Appl. Phys. Exp., 2009, 2, 121501.

[67] B. Rozic,V. Tzitzios, E. Karatairi, U. Tkalec, G. Nounesis, Z. Kutnjak, G. Cordoyiannis, R. Rosso, E. G. Virga, I. Musevic and S. Kralj, Eur. Phys. J. E, 2011, 34, 17.

[68] B. Senyuk, J. S. Evans, P. J. Ackerman, T. Lee, P. Manna, L. Vigderman, E. R. Zubarev, J. van de Lagemaat and I. I. Smalyukh, Nano Letters, 2012, 12,955.

[69] G. Cordoyiannis, V. S. R. Jampani, S. Kralj, S. Dhara, V. Tzitzios, G. Basina, G. Nounesis, Z. Kutnjak, C. S. P. Tripathi, P. Losada-Perez, D. Jesenek, C. Glorieux, I. Mu`sevi`c, A. Zidansek, H. Ameinitsch and J. Thoen, SoftMatter, 2013, 9, 3956.

[70] J.-C. Géminard, C. Laroche, P. Oswald, Phys. Rev. E, 1998, 58, 5923.

[71] J. Müller, J. M. Lupton, A. L. Rogach, J. Feldmann, D. V. Talapin and H. Weller, Phys. Rev. B, 2005, 72, 205339.

[72] K. Gueu, E. Megnassan and A. Proutiere, Mol. Cryst. Liq. Cryst.,1986, 132, 303. 